\title{
Simulation and quantitative analysis of fluorescence intensity distribution based on the Monte Carlo method
}

\author{
Kun Zhou*, Jian Tian*, Qiushi Zhang*, , Xiangxi Meng*, \\ Kun Yang* and Qiushi Ren**: \\ *Department of Biomedicine and Engineering \\ College of Engineering, Peking University \\ No. 5 Yiheyuan Road, Beijing 100871, P. R. China \\ ${ }^{\dagger}$ Institute for Drug and Instrument Control of PLA \\ Beijing, P. R. China \\ †renqsh@coe.pku.edu.cn
}

Received 16 January 2015

Accepted 13 April 2015

Published 22 May 2015

\begin{abstract}
The Monte Carlo method is a versatile simulation algorithm to model the propagation of photons inside the biological tissues. It has been applied to the reconstruction of the fluorescence molecular tomography (FMT). However, such method suffers from low computational efficiency, and the time consumption is not desirable. One way to solve this problem is to introduce a priori knowledge which will facilitate iterative convergence. We presented an in vivo simulation environment for fluorescence molecular tomography (ISEFMT) using the Monte Carlo method to simulate the photon distribution of fluorescent objects and their sectional view in any direction quantitatively. A series of simulation experiments were carried out on different phantoms each with two fluorescent volumes to investigate the relationship among fluorescence intensity distribution and the excitation photon number, the locations and sizes of the fluorescence volumes, and the anisotropy coefficient. A significant principle was discovered, that along the direction of the excitation light, the fluorescent volume near the excitation point will provide shelter effect so that the energy of the fluorescent volume farther away from the excitation point is relatively lower. Through quantitative analysis, it was discovered that both the photon energy distribution on every cross section and the fluorescence intensity distributed in the two volumes exhibit exponential relationships. The two maximum positions in this distribution correspond to the centers of fluorescent volumes. Finally, we also explored the effect of the phantom coefficients on the exponential rule, and found out that the exponential rule still exists, only the coefficient of the exponential rule changed. Such results can be utilized in locating the positions of multiple fluorescent volumes in complicated FMT reconstruction involving multiple fluorescent volumes. Thus, a priori knowledge can be generalized, which would accelerate the reconstruction of FMT and even other images.
\end{abstract}

Keywords: Monte Carlo simulation; FMT; quantitative.

\$Corresponding author.

This is an Open Access article published by World Scientific Publishing Company. It is distributed under the terms of the Creative Commons Attribution 3.0 (CC-BY) License. Further distribution of this work is permitted, provided the original work is properly cited. 


\section{Introduction}

Fluorescence imaging technology has been translated into a potent tool in medical diagnostics. ${ }^{1}$ The development of biotechnologies, especially genetic engineering and molecular fluorescence technologies, enabled the revolutionary technology of fluorescence molecular tomography (FMT). Such technology works with near-infrared (NIR) light $(600-900 \mathrm{~nm})$ which demonstrates the best tissue penetration property, and provides information about the three-dimensional distribution of molecular (or nano-) fluorescent probes, thus can reveal molecular processes in vivo. ${ }^{2}$ This marks the evolution of tissue optical imaging technology from the organ-level to the molecular and cellular levels. ${ }^{3}$ With a proper design of molecular probes, FMT can realize in vivo detection of molecular and cellular events such as gene expression, tumor angiogenesis and cancer cell proliferation. ${ }^{4-6}$ To date, FMT systems have evolved from the early fiberbased systems ${ }^{7}$ to noncontact slab-shaped systems using charge coupled devices (CCD) ${ }^{8}$ Owing to the reasons stated above, the FMT reconstruction algorithms are becoming an active area of research. In recent years, rapid progress has been made in the reconstruction of FMT images, and algorithms, such as finite-element-based methods, finite difference methods and Monte Carlo methods are being extensively studied. ${ }^{9}$

The Monte Carlo simulation is an important tool in biomedical optics. ${ }^{10,11}$ Albeit restrictions on the application of such a method is relatively minimal even in complex media, it suffers from poor efficiency. ${ }^{9}$ Kumar et al. ${ }^{12}$ proposed a reconstruction strategy based on a Monte Carlo method that reconstructs the distribution of the fluorescence lifetime in a time-domain fluorescence tomography for small-animal imaging. A phantom experiment demonstrated that this method can clearly distinguish two fluorescent point sources as close as $6 \mathrm{~mm}$ apart. Zhang et al. ${ }^{13}$ also used a Monte Carlo method in the reconstruction of fluorescence tomography for small-animal imaging. In their research, nevertheless, it usually took no less than $6 \mathrm{~h}$ to run the reconstruction program, owing to the poor computational efficiency and the great amount of Green functions that slowed down the calculation. ${ }^{14}$

It will greatly facilitate the iterative convergence of the Monte Carlo methods if a priori knowledge about the fluorescent photon distribution (especially the centers of different fluorescent volumes) could be obtained in advance of the reconstruction. Here we reasonably assumed that there is a quantitative pattern of the exited fluorescent photon distribution in the case of the FMT. If the assumption proved correct, this semi-empirical pattern can be employed in the optimization of the initial guesses and the acceleration of iterations by providing a priori knowledge in Monte Carlo and even other FMT reconstruction techniques. Such reasoning is based on the experience with previously available Monte Carlo platforms, such as Monte Carlo simulations for multi-layered tissues (MCML) ${ }^{11}$ which accurately simulates the scattering of photons in multi-layered tissue, and mouse optical simulation environment (MOSE), ${ }^{15}$ which focuses on bioluminescence imaging.

To study the photon distribution pattern, we developed an in vivo simulation environment for fluorescence molecular tomography (ISEFMT) which uses the Monte Carlo method to predict the threedimensional photon distribution of the fluorescent biomarkers and their sectional view in any direction. In this paper, ISEFMT is first evaluated, and its stability and validity has been confirmed. Then, a series of experiments are presented to reveal the relationship among the fluorescence intensity distribution, the excitation photon number, the location of the fluorescence biomarker, the size of the fluorescent volume and the anisotropy coefficient. This illustrated some principles with straightforward images. In the simulation of a model phantom consisting two spherical fluorescent volumes, we found a special "shelter phenomenon". Quantitative analysis was conducted to explain such phenomenon, and an exponential law was formularized. Finally, a series of experiments on the effect of the phantom coefficients on the exponential law are presented, and this revealed that they only affect coefficients of the exponential formula while the general form is consistent. Having concluded and quantitatively described such phenomena, our work provided a new possibility to accelerate the reconstruction of FMT images with a priori knowledge. An attenuation correction database could be subsequently created based on our work, and it could be applied in situations with great complexity. 


\section{Theory}

\subsection{Photon propagation}

The process of fluorescence photon propagation in the tissue is as follows. First, excitation photons enter the tissue and propagates inside. When they enter a fluorescent volume, fluorescence photons will be generated. Subsequently, fluorescence photons spread in the tissue, and a portion of them will penetrate through the tissue surface and, subsequently, be detected. Note that the light propagation is influenced by the property of the tissue. Different kinds of biological tissues can be defined by their optical and geometric parameters. ${ }^{15}$

The light beam of the external light source is assumed to be a pencil beam. A certain number of photons are treated as a package with an initial weight, which corresponds to a dimensionless energy. During the light propagation in tissues, the weight of light decreases due to the interaction between the photon package and the tissue. Biological tissues have a strong scattering effect versus absorption on the light beam. ${ }^{16,17}$ Photon scattering refers to the direction change after collision. In photon propagation, the displacement of a package is determined by two parameters: step size (the optical distance that the packages travel during the time elapsed) and direction.

The photon step size $S$, direction $\varphi$ and $\theta$ can be obtained as follows (Eqs. (1)-(3)). ${ }^{15,18}$

$$
\begin{gathered}
S=\frac{-\ln \xi}{u_{a}+u_{s}}, \\
\cos \theta= \begin{cases}\frac{1}{2 g}\left\{1+g^{2}-\left(\frac{1-g^{2}}{1-g+2 g \xi}\right)^{2}\right\} & \begin{array}{l}
\text { if } g \neq 0 \\
2 \zeta-1
\end{array} \\
\text { others }\end{cases}
\end{gathered}
$$

$$
\varphi=2 \pi \zeta_{\varphi}
$$

where $\xi$ and $\zeta_{\varphi}$ are different random numbers between 0 and $1 ; u_{a}$ is the absorption coefficient; $u_{s}$ is the scattering coefficient; $\varphi$ and $\theta$ are azimuth angle and deflection angle after scattering, respectively; and $g$ is the anisotropy factor.

As shown in Fig. 1, when the photons travel to the interface of two layers of tissue differing in their refraction index $n$, they are reflected and, sometimes, also refracted. In the case of refraction, the photons penetrate through the interface and continue to propagate in the other layer of tissue; whereas in the case of reflection, the photons

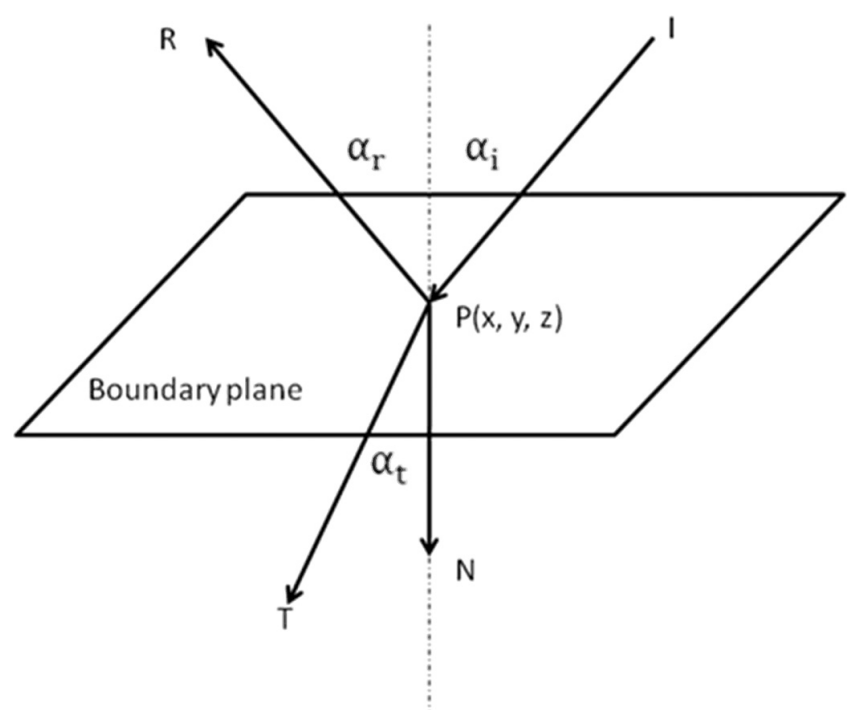

Fig. 1. The reflection and refraction of light.

continue to spread in the original layer. The probability of reflection $R\left(a_{i}\right)$ is calculated with the following equations (Eqs. (4)-(6):

$$
\begin{aligned}
& R\left(\alpha_{i}\right)=\frac{1}{2}\left[\frac{\sin ^{2}\left(\alpha_{i}-\alpha_{t}\right)}{\sin ^{2}\left(\alpha_{i}+\alpha_{t}\right)}+\frac{\tan ^{2}\left(\alpha_{i}-\alpha_{t}\right)}{\tan ^{2}\left(\alpha_{i}+\alpha_{t}\right)}\right], \\
& \vec{U}=\vec{I}-2(\vec{I} \bullet \vec{N}) \vec{N}, \\
& \vec{U}=\sin \alpha_{t} \bullet \frac{\vec{I}-(\vec{I} \bullet \vec{N}) \vec{N}}{|\vec{I}-(\vec{I} \bullet \vec{N}) \vec{N}|}+\operatorname{sign}(\vec{I} \bullet \vec{N}) \\
& \quad \times \cos \alpha_{t} \vec{N},
\end{aligned}
$$

where $a_{i}$ and $a_{t}$ are the angle of incidence and angle of refraction, respectively; $\vec{I}$ is the direction vector of the incident light; $\vec{N}$ is the normal vector and $\vec{U}$ is the new photon direction after reflection or refraction. If reflection occurs, should be determined by Eq. (5); otherwise, $\vec{U}$ should be determined by Eq. (6).

When the excitation light hits the fluorescence volumes, fluorescence photons will be generated. The intensity of the fluorescence is determined by the excitation light intensity and the quantum yield of the fluorescence biomarkers. The fluorescence intensity $I_{f}$ can be calculated as ((Eq. (7)):

$$
I_{f}=I_{\varepsilon} \times a \times p,
$$

where $I_{\varepsilon}$ is the intensity of the excitation light; $a$ is the absorption coefficient (which means the probability of absorption) of the fluorescence volume; and $p$ is the quantum yield of the fluorescence biomarker. 


\subsection{Simulation realization}

Based on the above-mentioned process, a new Monte Carlo simulation program was coded in $\mathrm{C}++$ language. It can simulate the intensity distribution of fluorescent photons in any cross section as well as on the surface of our model. Figure 2 shows the transmission process of the photon packages. Firstly, the program will initialize all of the parameters of the simulation model as well as the property of the excitation photons package (the weight or energy of each photon package is set as 1).
Then, the photon package will move on according to the step parameters determined by the random process. This is realized through loop structure called "propagation loop". Subsequently, the program will judge whether the photon package enters the fluorescent volume or exits the phantom, and in both cases the propagation rules alter. In the former case, if the excitation photon package propagates in the fluorescent volume, it will generate fluorescent photons, and the program enters the excitation loop. After jumping out of the excitation loop, the

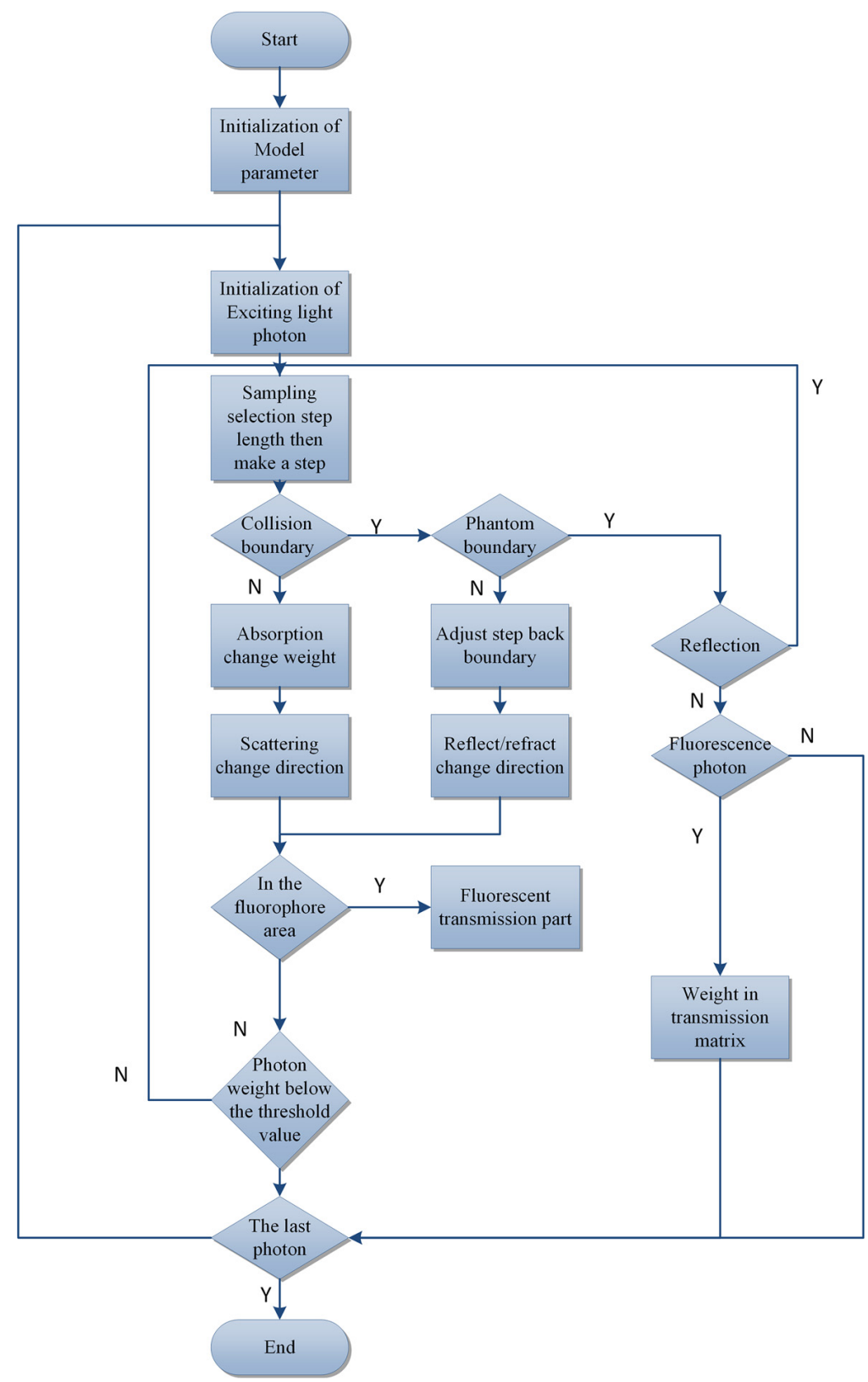

Fig. 2. Flowchart of ISEFMT with the Monte Carlo method. 
program will return to the propagation loop. In the latter case, if the photon package exits the phantom, the propagation loop terminates, and a new photon package is initialized, and then a new propagation loop starts.

In our program we designed three classes so that the simulation model could be adapted conveniently. The parent class, named "Tissue" retains all of the model parameters such as the absorption coefficients as its variables; two child classes (the "Ellipsoid" and "Cylinder" classes) contain functions that simulate the transmission of photon packages. Functions defined in the class "Tissue" are used to set or get the value of its variables. In class Ellipsoid, several functions are defined to control or simulate the transmission of photon packages, and the same holds true for the class "Cylinder". As a result, the simulation model can be defined conveniently by tuning the corresponding class object.

\section{Experimental Methods}

The phantom being studied is shown in Fig. 3. It was a cylinder representing the normal biological tissue with two ellipsoidal fluorescent volumes inside (for simplicity, we only tested spheres as a special case in this paper, of which the size parameters were three repeated numbers denoting their radii). A Cartesian coordinate was defined, with the origin at the geometric center of the cylinder and $Z$-axis parallel to its axis. As shown in Table 1, optical parameters of the normal tissue were invariable throughout our work, while the parameters of the fluorescent volumes (the sphere in Fig. 3) were constant unless otherwise specified. The incidence of the excitation light $(500 \mathrm{~nm})$ was vertical and occurred at the center of the upper face of the cylinder.

\subsection{Platform validation}

In the repeated simulation validation experiment, 10 parallel simulations on the photon distribution of the phantom with the same set of parameters were performed. The positions of the two spherical fluorescent volume were set as $(-2,-2,0)$ and $(4,4,0)$, and the number of photons in each simulation was $10^{6}$, which is the least number of photons to guarantee the accuracy of the simulation. The total

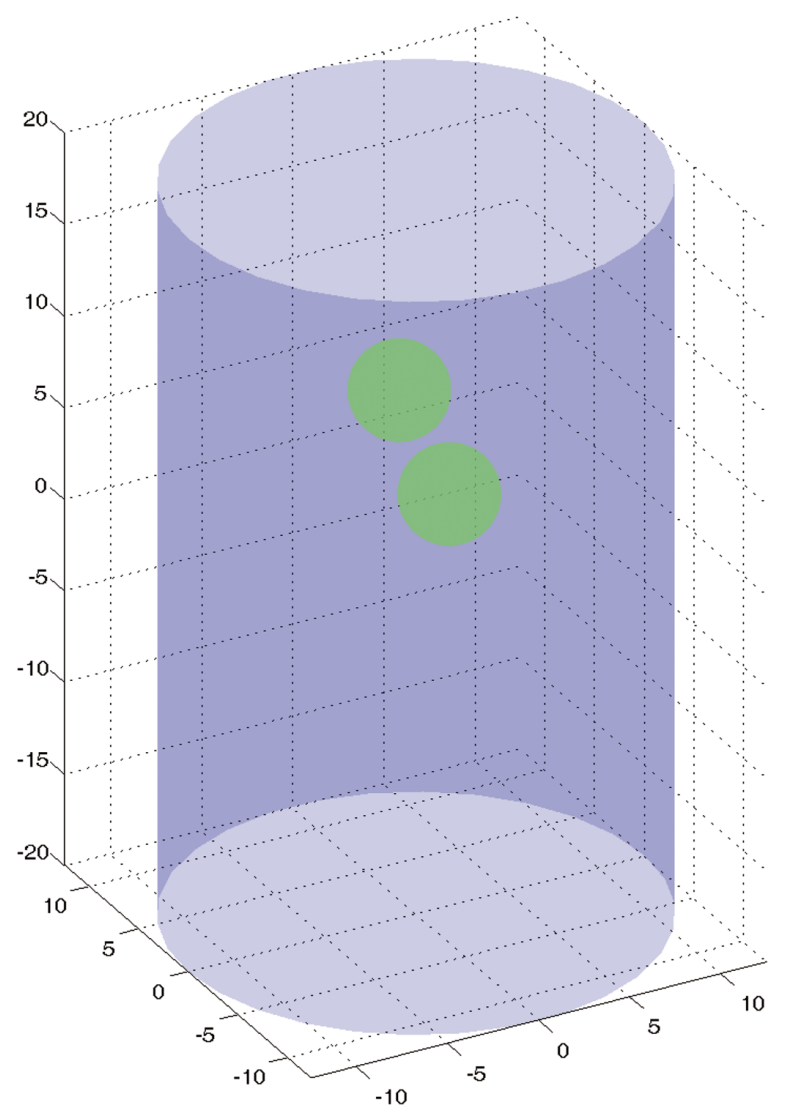

Fig. 3. The simulation mode.

photon energy of selected transaxial slices was chosen as the metric to evaluate the stability of the platform. Six slices were selected, and their $z$ coordinates were $z=18,10,6,0,-10$ and -18 . The total energy was defined as the summation of the energy of the photons distributed in the given slice. A total of 10 simulated total energy values were given for each slice, and their coefficients of variation $(\mathrm{CV})$ were calculated.

Table 1. Parameters of the normal tissue.

\begin{tabular}{|c|c|c|c|}
\hline \multicolumn{2}{|l|}{$\begin{array}{l}\text { Parameters for } \\
\text { the cylinder }\end{array}$} & \multicolumn{2}{|c|}{$\begin{array}{l}\text { Parameters for the } \\
\text { fluorescent volumes }\end{array}$} \\
\hline $\begin{array}{l}\text { Size parameters } \\
\quad(\mathrm{mm})\end{array}$ & $(12.5,12.5,40)$ & $\begin{array}{l}\text { Size parameters } \\
\quad(\mathrm{mm})\end{array}$ & $(2.5,2.5,2.5)$ \\
\hline$\mu_{a}\left(\mathrm{~mm}^{-1}\right)$ & 0.03 & $\mu_{a}\left(\mathrm{~mm}^{-1}\right)$ & 0.01 \\
\hline$\mu_{s}\left(\mathrm{~mm}^{-1}\right)$ & 10.00 & $\mu_{s}\left(\mathrm{~mm}^{-1}\right)$ & 10.00 \\
\hline $\begin{array}{l}\text { Anisotropy } \\
\text { coefficient }\end{array}$ & 0.9 & $\begin{array}{l}\text { Anisotropy } \\
\text { coefficient }\end{array}$ & 0.9 \\
\hline \multirow[t]{2}{*}{$\begin{array}{l}\text { Refraction } \\
\text { coefficient }\end{array}$} & 1.4 & $\begin{array}{l}\text { Refraction } \\
\text { coefficient }\end{array}$ & 1.2 \\
\hline & & $\begin{array}{l}\text { Quantum } \\
\text { efficiency }\end{array}$ & 0.1 \\
\hline
\end{tabular}




\section{K. Zhou et al.}

In the cross-validation experiment, two parallel simulations of the same model in MOSE (provided by Jie Tian et al.) and ISEFMT program were performed, so as to evaluate the validity of our adapted method. The phantom parameters were the same as in the previous repeated simulation experiment. A curve of photon energy distribution in transaxial slices along the $z$-direction was generated. A curve of the photon energy distribution along the orthogonal orientations traversing the center of the fluorescent volume within a certain cross section was also obtained. With such information, the coincidence of the simulation results could be measured, which demonstrated the quality and accuracy of our approach.

\subsection{Principles of fluorescence intensity distribution}

A series of simulation experiments were performed on a simplified phantom with only one spherical fluorescent volume to reveal the relationship among the fluorescence intensity distribution, the excitation photon number, the location of the fluorescence biomarker, the size of the fluorescent volume, and the anisotropy coefficient. These relationships could be obtained through variable-control simulations with only one parameter altered each time.

\subsection{Fluorescence intensity distribution of two spherical fluorescent volumes}

To study the photon distribution in the phantom with two spherical fluorescent volumes, a preliminary experiment was designed. In this experiment, each simulation was conducted with 5000 excitation photons. Two simulations with the fluorescent volume positions of $(-2,-2,0),(4,4,0)$ and $(4,4,0)$, $(-2,-2,10)$ were carried out in this experiment.

\subsection{Quantitative analysis of shelter effect}

In order to quantitatively explain the special characteristics in the intensity distribution of the fluorescence photons, a series of simulation experiments were designed using $10^{6}$ excitation photons. The positions of one fluorescent volume was set constant (expect in the first simulation), while the other
Table 2. Experiment parameter of the changed fluorescent volume.

\begin{tabular}{cc}
\hline Simulation number & Ellipsoid origin coordinates $(\mathrm{mm})$ \\
\hline 1 & $(-2,-2,0)(4,4,0)$ \\
2 & $(-2,-2,10)(4,4,2)$ \\
3 & $(-2,-2,10)(4,4,6)$ \\
4 & $(-2,-2,10)(4,4,10)$ \\
5 & $(-2,-2,10)(4,4,-6)$ \\
\hline
\end{tabular}

varied along $z$-axis, as listed in Table 2. The total energy of fluorescent photons on each cross section was obtained after each simulation, with the step length of $2 \mathrm{~mm}$.

In order to quantitatively study the relationship between the energy distribution and the positions of the fluorescent volumes, the following experiment was conducted. A total of nine simulations were performed with the fluorescent volume positions listed in Table 3. A new variable $R$ was introduced, and was defined as:

$$
R=\frac{L 1}{L 2},
$$

where $L 1$ and $L 2$ are distances between the center of fluorescent volumes and the elongated excitation photons incident beam.

In order to explore the influence of altering various physical parameters of the phantom on photon distribution, a series of simulations were conducted. The positions of the two fluorescent volumes were set as $(-2,-2,10)$ and $(3.2,3.2,-5)$, respectively, and the size parameters of the two ellipsoids were set as $(1.5,1.5,1.5)$ to save simulation time. In each simulation group, one of the parameters changed while all others were kept constant among different

Table 3. Experiment parameter of the two fluorescent volumes.

\begin{tabular}{cll}
\hline Simulation number & Ellipsoid origin coordinates $(\mathrm{mm})$ & $R$ \\
\hline 1 & $(-2,-2,10)(0.8,0.8,10)$ & 0.4 \\
2 & $(-2,-2,10)(1.2,1.2,10)$ & 0.6 \\
3 & $(-2,-2,10)(1.6,1.6,10)$ & 0.8 \\
4 & $(-2,-2,10)(2,2,10)$ & 1 \\
5 & $(-2,-2,10)(2.4,2.4,10)$ & 1.2 \\
6 & $(-2,-2,10)(2.8,2.8,10)$ & 1.4 \\
7 & $(-2,-2,10)(3.2,3.2,10)$ & 1.6 \\
8 & $(-2,-2,10)(3.6,3.6,10)$ & 2 \\
9 & $(-2,-2,10)(4,4,10)$ & \\
\hline
\end{tabular}


Table 4. Changed parameters of the experiment.

\begin{tabular}{clr}
\hline Simulation group number & \multicolumn{1}{c}{ Changed parameters } & \multicolumn{1}{c}{ Values } \\
\hline 1 & Phantom's refraction coefficient $n$ & $1.4 ; 1.6 ; 1.8 ; 2.0 ; 2.2 ; 2.4$ \\
2 & Phantom's anisotropy coefficient $g$ & $0.3 ; 0.5 ; 0.7 ; 0.9$ \\
3 & Fluorescent volume's refraction coefficient $n$ & $1.4 ; 1.6 ; 1.8 ; 2.0 ; 2.2 ; 2.4$ \\
4 & Fluorescent volume's anisotropy coefficient $g$ & $0.3 ; 0.5 ; 0.7 ; 0.9$ \\
5 & Upper fluorescent volume size $R$ & $2.0 ; 2.5 ; 3.0$ \\
6 & Lower fluorescent volume size $R$ & $2 ; 2.5 ; 3 ; 3.5$ \\
\hline
\end{tabular}

simulations as in Table 1. The changed parameters are listed in Table 4.

\section{Result and Discussion}

\subsection{Establishment of method and preliminary results}

The stability as well as credibility of the new simulation platform, ISEFMT, was demonstrated through repeated calculations and cross-validation with another benchmark method.

Although Monte Carlo is a probability-based algorithm, it should generate reproducible results from same sets of input conditions. In the repeated simulating experiment, the energy distribution of the model was repeatedly calculated for 10 times. The total energy of seven different transaxial slices from each simulation result was compared statistically and their characteristics are listed in Table 5. This analysis suggests that the ISEFMT system is sufficiently robust, as the CV of the total energy in each slice are all very small (not more than 0.0089 ).

At present, MOSE is one of the most comprehensive software platform in FMT simulations, so it was used as a benchmark method to evaluate our platform performance. In the cross-validation experiment, a same model was simulated in both platforms (shown in Fig. 4). Figure 4(c) shows the photon intensity along an arbitrary line in a transaxial cross section. It is evident that the fluorescence intensity distribution trends obtained from both methods were almost identical. Moreover, with the same excitation photon number, the curve given by ISEFMT seemed to be smoother than that by MOSE which may suggest better data distribution continuity.

With the above two experiments, it is concluded that the ISEFMT platform is capable of generating stable and reliable photon distribution, and is in some aspects superior to the previous programs.

Schematic and qualitative pattern of fluorescent photon distribution was obtained with a simplified phantom with only one fluorescent volume. This simplification avoided the nonlinear distribution thus could imply a general law of photon behavior. Figure 5 shows the relationship among the photon number, fluorescent volume location, fluorescent volume size, and anisotropy coefficient. When the photon number or the fluorescent volume size was increased, the energy distribution became smoother

Table 5. Repeated simulation results.

\begin{tabular}{lccccccc}
\hline & $Z=18$ & $Z=10$ & $Z=6$ & $Z=0$ & $Z=-6$ & $Z=-10$ & $Z=-18$ \\
\hline Simulation 1 & 0.00430044 & 0.0182087 & 0.00466489 & 0.00307545 & 0.00214746 & 0.00171105 & 0.00118121 \\
Simulation 2 & 0.00431153 & 0.0183325 & 0.00475485 & 0.00305732 & 0.00212814 & 0.00171153 & 0.00117242 \\
Simulation 3 & 0.00425044 & 0.0182835 & 0.00473500 & 0.00302061 & 0.00214015 & 0.00174203 & 0.00118088 \\
Simulation 4 & 0.00428122 & 0.0183558 & 0.00471083 & 0.00302295 & 0.00211633 & 0.00172314 & 0.00117588 \\
Simulation 5 & 0.00426450 & 0.0182503 & 0.00471982 & 0.00308486 & 0.00215626 & 0.00172479 & 0.00118130 \\
Simulation 6 & 0.00431240 & 0.0183496 & 0.00468569 & 0.00305662 & 0.00215493 & 0.00172698 & 0.00117583 \\
Simulation 7 & 0.00429630 & 0.0182994 & 0.00470785 & 0.00304599 & 0.00215183 & 0.00170957 & 0.00118521 \\
Simulation 8 & 0.00428556 & 0.0182608 & 0.00475827 & 0.00305516 & 0.00212398 & 0.00174159 & 0.00117470 \\
Simulation 9 & 0.00432001 & 0.0182224 & 0.00471665 & 0.00303516 & 0.00214066 & 0.00173877 & 0.00119963 \\
Simulation 10 & 0.00431413 & 0.0182087 & 0.00471408 & 0.00303756 & 0.00211207 & 0.00174929 & 0.00115863 \\
Mean & 0.00429365 & 0.0182771 & 0.00471679 & 0.00304917 & 0.00213718 & 0.00172787 & 0.00117857 \\
S.D. & $2.30348 \mathrm{e}-5$ & $5.61648 \mathrm{e}-5$ & $2.84682 \mathrm{e}-5$ & $2.09920 \mathrm{e}-5$ & $1.61290 \mathrm{e}-5$ & $1.44674 \mathrm{e}-5$ & $1.03962 \mathrm{e}-5$ \\
C.V. & 0.00536486 & 0.00307295 & 0.0060355 & 0.00688450 & 0.00754687 & 0.00837297 & 0.00882103 \\
\hline
\end{tabular}




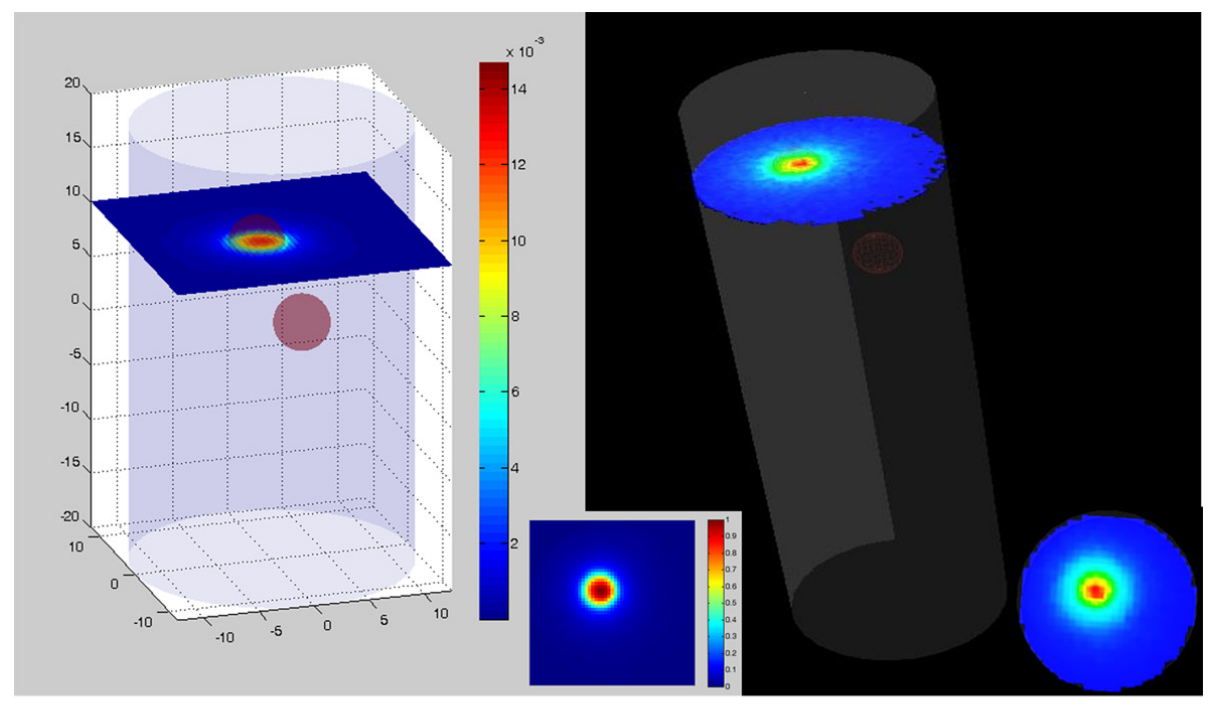

(a)

(b)

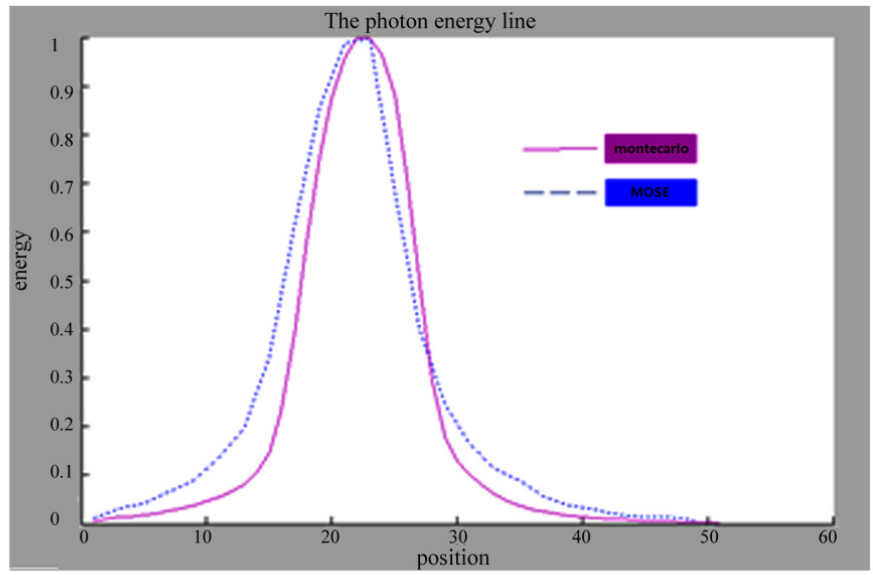

(c)

Fig. 4. Comparison between ISEFMT and MOSE visually and quantitatively through the photon energy trend line. (a) The simulation by ISEFMT, (b) the simulation by MOSE and (c) comparison between ISEFMT and MOSE.

(Figs. 5(a) and 5(c)). When the fluorescent volume was deeper, the fluorescence intensity decreased ((Fig. 5(b)). When the anisotropy coefficient (ranging from 0 to 1$)$ increased, more fluorescence photons were generated $(($ Fig. $5(\mathrm{~d}))$; this is because when the anisotropy coefficient increases, the probability of the photons reaching the fluorescent volume increases. As shown in Fig. 4(e), we can clearly demonstrate the information of the sectional view in any direction.

\subsection{Qualitative and quantitative pattern of photon distribution}

The attenuation of light intensity by absorption and scattering can be quantitatively described by
Lambert-Beer's law. However, if two or more light sources are involved, the situation cannot be depicted directly and a numerical approach is thus adopted. Owing to the interplay between the light sources, an adjusted correlation should be obtained. In this paper, the representative situation involving two fluorescent volumes is discussed, using the Monte Carlo platform we proposed, to aid the reconstruction of more complex fluorescent images.

In the qualitative demonstration experiment, the photon distribution pattern of the two-fluorescent volume phantom was obtained with ISEFMT platform (shown in Fig. 6). With a close scrutiny on this result, a preliminary conclusion can be drawn that the fluorescent volumes in higher positions will generate more fluorescent photons than those in 


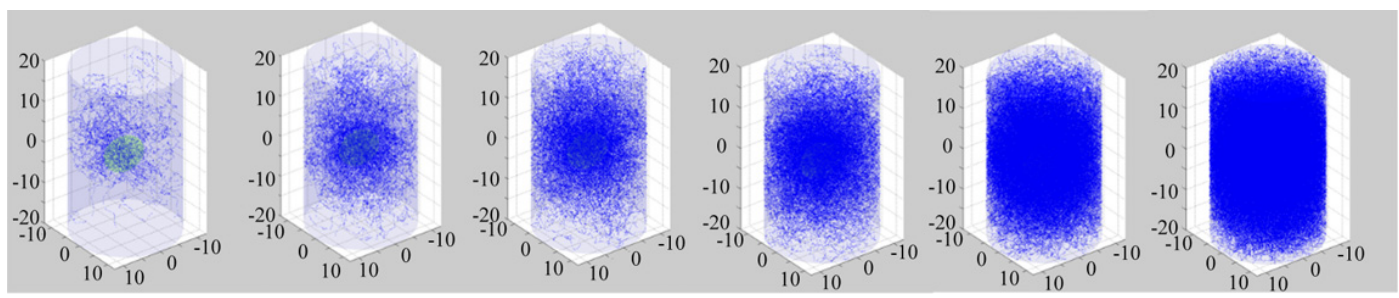

(a)

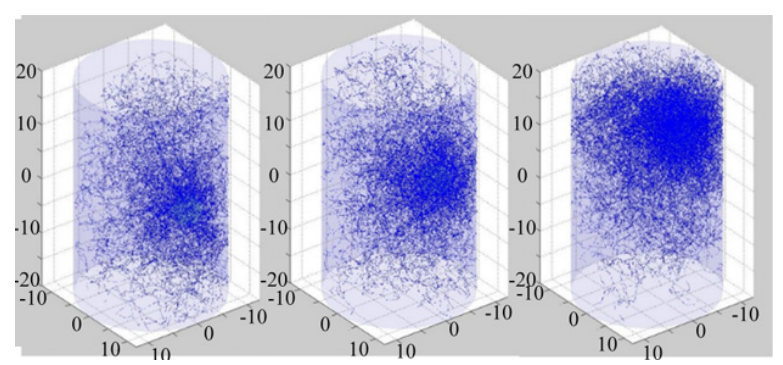

(b)

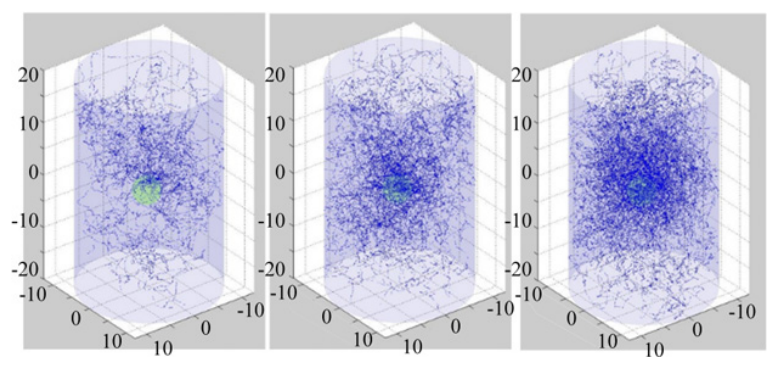

(d)
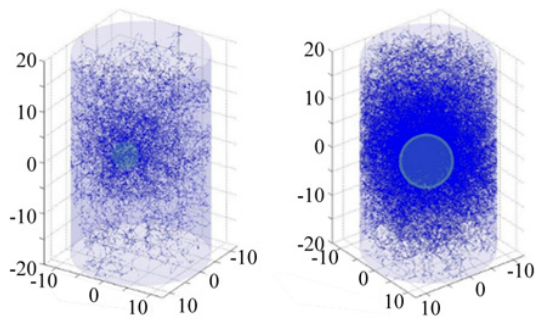

(c)

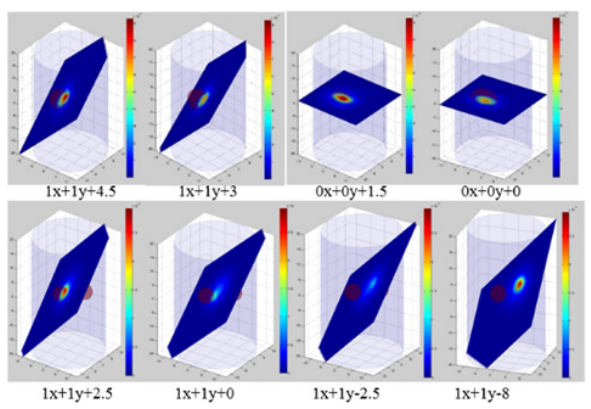

(e)

Fig. 5. A series of simulation experiments about fluorescence intensity distribution. (a) The relationship between the fluorescence intensity distribution and the excitation photon number. The excitation photon numbers are 100, 500, 1000, 2000, 5000 and 10,000, respectively. (b) The relationship between the fluorescence intensity distribution and the location of the fluorescence volumes. Coordinates are $(5,-3,-5),(5,-3,0)$ and $(5,-3,9)$, respectively. (c) The relationship between the fluorescence intensity distribution and the size of the fluorescent volume, size of spherical areas are $(2.5,2),(5,2.5)$, and $(5,5,5)$ respectively. (d) The relationship between the fluorescence intensity distribution and the anisotropy coefficient. The values of are $0.3,0.6$, and 0.9 , respectively. (e) Sectional view from various directions.

lower positions [compare Figs. 6(a), 6(d) and 6(f)], in other words, along the direction of the initial excitation light, the fluorescent volume near the excitation point will provide shelter so that the energy of the fluorescent volume which is further away from the excitation point is relatively low. This is because the fluorescent volumes closer to the incident point will have higher probabilities of catching the excitation photons.

A quantitative summary was established from the quantitative analysis experiment. Figure 7 demonstrates the photon energy distribution on every cross section. The abscissa means the cross section position (because it is a plane parallel to the $X-Y$ plane, so it is presented just with $Z$ coordinate value) and the longitudinal coordinate means the total energy in the cross section. It can be concluded that the total energy contour of each cross section reached the highest level at the center of the fluorescent volume, and then attenuated in both directions following the exponential law: the total energy in each cross section shows a piecewise exponential relationship with the cross section position (coordinate $z$ ). In Fig. 8, the abscissa axis represents the ratio of distance between the two fluorescent volumes to the excitation photon; whereas, the ordinate represents the ratio of 
K. Zhou et al.

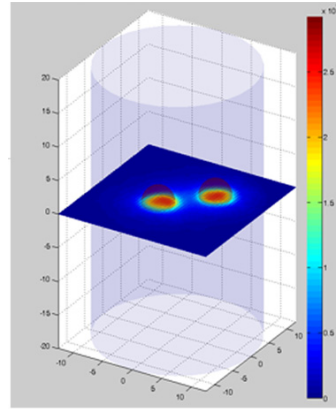

(a) the energy distribution on the $\operatorname{plan}(\mathrm{z}=0)$

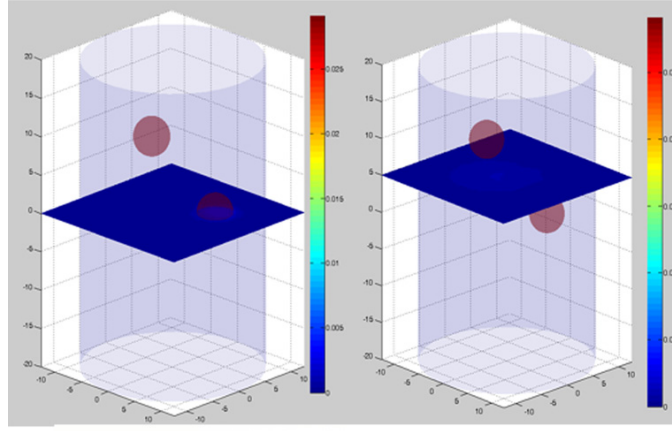

(d) the energy distribution on the $\operatorname{plan}(\mathrm{z}=0)$ (e) the energy distribution on the $\operatorname{plan}(\mathrm{z}=5)$

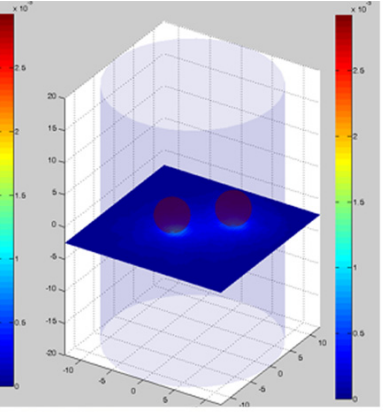

(c) the energy distribution on the $\operatorname{plan}(\mathrm{z}=-2)$

Fig. 6. Fluorescence intensity distribution of two spherical foci.

\section{Photon energy distribution on cross section}

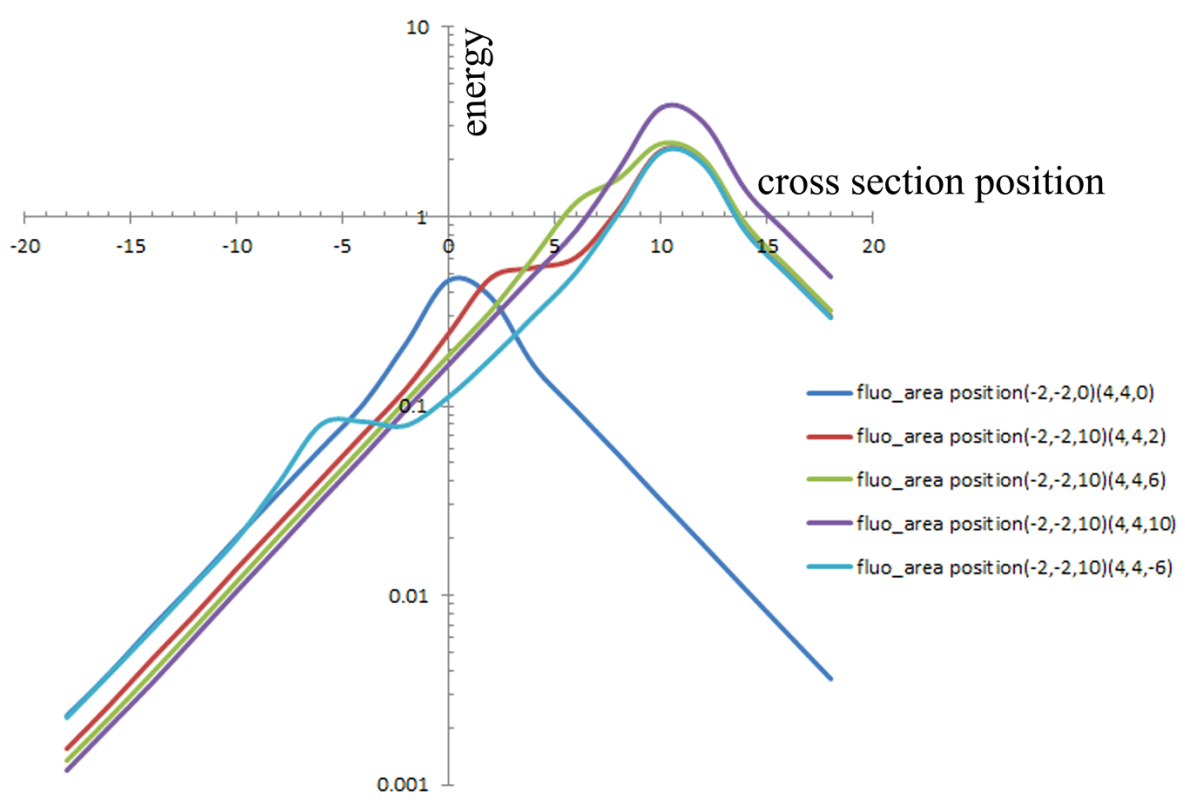

Fig. 7. Photon energy distribution on cross section. 


\section{The energy ratio of two fluorescent areas}

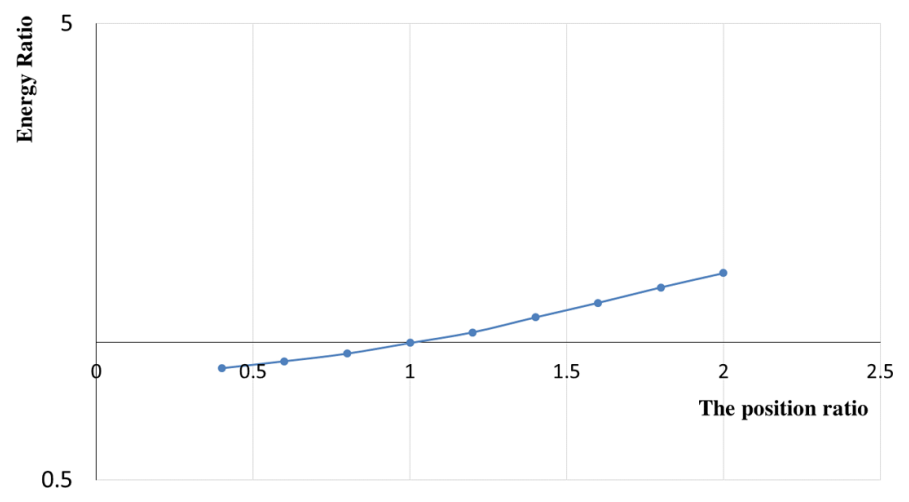

Fig. 8. Energy ratio of two fluorescent volumes.

the total energy of the two fluorescent volumes. This figure shows that the energy ratio between the two fluorescent volumes followed the exponential distribution as well. Since both the photon energy distribution on every cross section and the fluorescence intensity distribution in the two volumes met the exponential relationship, we could build a model to delineate the quantitative relationship, namely ((Eq. (9) or Eq. (10)):

$$
E_{\mathrm{tot}, i}=A \times \mathrm{e}^{c z_{i}},
$$

$E_{\text {tot }, i}$ : Total energy in each cross section $i$.

$z_{i}$ : Position of cross section $i$.

$A, c$ : The constant coefficients to be determined.

$$
\frac{E_{T 1}}{E_{T 2}}=B \times \mathrm{e}^{d \times R a}
$$

$E_{T 1}$ : Total energy in fluorescent volume 1 , $E_{T 2}$ : Total energy in fluorescent volume 2 ,

$R a$ : The distance ratio between the two fluorescent volumes. Defined in Eq. (8).

$B, d$ : The constant coefficients to be determined.

For example, we chose the results obtained from simulation 5 in the qualitative analysis experiment (as in row 5 of Table 2), fitted them with the expression ((Eq. (9)) above. The piecewise results are as follows:

$$
\begin{aligned}
& \hat{E}_{\mathrm{tot}}=0.4165 \mathrm{e}^{0.294 z}, \quad R^{2}=0.9961 ; \quad(-18<z<-6), \\
& \hat{E}_{\mathrm{tot}}=0.0771 \mathrm{e}^{0.007 z}, \quad R^{2}=0.9349 ; \quad(-6<z<-2), \\
& \hat{E}_{\mathrm{tot}}=0.1128 \mathrm{e}^{0.2769 z}, \quad R^{2}=0.9857 ;(-2<z<-10), \\
& \hat{E}_{\text {tot }}=37.468 \mathrm{e}^{-0.269 z}, \quad R^{2}=0.9721 ;(10<z<18),
\end{aligned}
$$

where $\hat{E}_{\text {tot }}$ is the total energy in each cross section while $z$ is the cross section position.
In addition, the result obtained from experiment (Table 3) is also fitted with expression 10, the formulation is shown as follows:

$$
\frac{E_{T 1}}{E_{T 2}}=0.7749 \mathrm{e}^{0.2863 z}, \quad R^{2}=0.9731,
$$

where $E_{T 1}$ is the total energy of the fluorescent volume 1 whose position is changed during the experiment. $E_{T 1}$ is the total energy of the fluorescent volume 2 located in $(-2,-2,10)$ as shown in Table 3 . $R a$ is the distance ratio defined in Eq. (8) and ranged from 0.4 to 2 during the experiment, as shown in Table 3.

This equation implies that the general trend of fluorescent energy distribution in such situations still follows the Lambert-Beer's law. However, due to the cross talk among multiple fluorescent volumes, the energy distribution demonstrates what is called a "shelter effect". Instead of a single-peak curve, a curve with multiple maximum points (each corresponding to the position of one fluorescent volume, i.e., the peak positions are at the centers of fluorescent volumes) will be generated. By employing this equation, the centers of fluorescent volumes can be easily located, which provides a general information of photon distribution.

The sensitivity of this model to various parameters were also tested, and the results are illustrated in Fig. 9. From all the figures we can conclude that the above-mentioned exponential law always existed in the situations simulated, which meant that the parameters did not change the exponential tendency. However, it did have an effect on the fluorescence intensity values. For instance, (A) indicates that as the normal tissue (cylinder) 


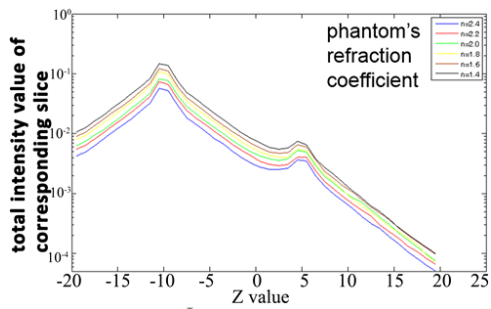

(a)

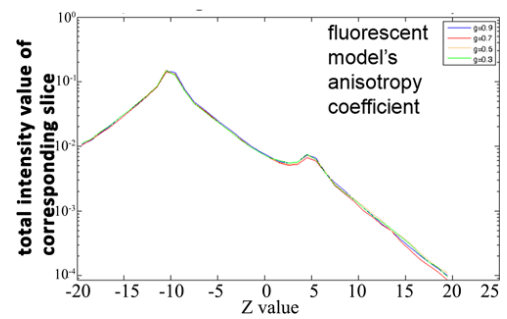

(d)

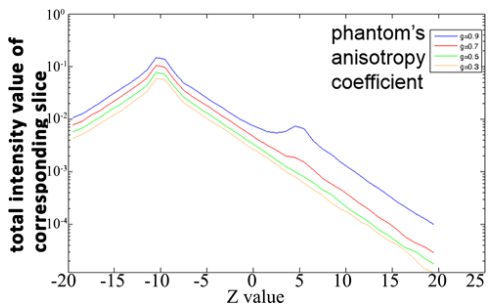

(b)

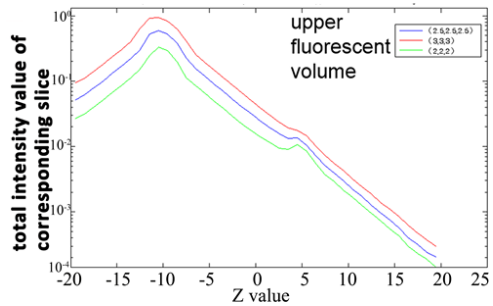

(e)

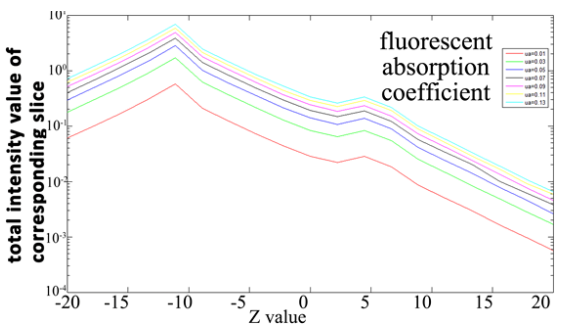

(g)

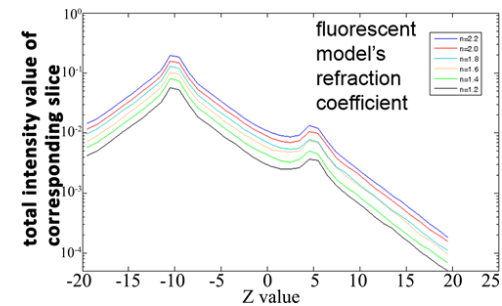

(c)

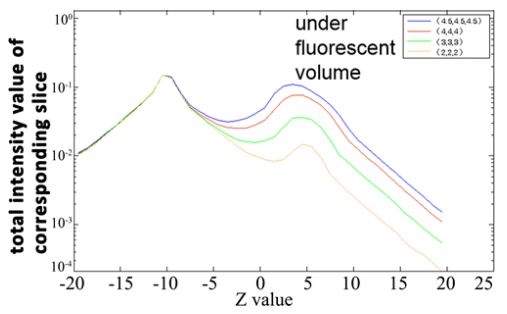

(f)

Fig. 9. Exponential law of total fluorescence intensity in each slice. (a) when the phantom's refraction coefficient changes, (b) when the phantom's anisotropy coefficient changes, (c) when the fluorescent volume's refraction coefficient changes, (d) when the fluorescent model's anisotropy coefficient changes, (e) when the higher fluorescent volume changes, (f) when the lower fluorescent volume changes and $(\mathrm{g})$ when the absorption coefficient changes.

refraction coefficient increased, the curve shifted down integrally, which meant that the total intensity decreased. This is because, along with the increase of the normal tissue refraction coefficient, the excitation photon would have a lower probability of entering the fluorescent volumes because of total reflection. (B) shows the effect of altering the normal tissue anisotropy coefficient. As the anisotropy coefficient increased, the excitation photons had a higher probability of getting deeper inside the whole phantom, which resulted in the increase of the fluorescence intensity near the lower fluorescent volume, as shown in (B). (C) implies a similar effect to (A), and they have the same explanation, as well. Along with the increase of the fluorescent volumes refraction coefficient, the possibility of the excitation photons entering the fluorescent volumes also increased, which resulted in an upward shift in (C).
From (D), we can conclude that the fluorescent volumes anisotropy coefficient had little effect on the exponential law; this might result from the small size of the fluorescent volumes. The relative size also had an effect on the exponential law, as shown by (E) and (F). As the size of one fluorescent volume increased, the fluorescence intensity near the volume also increased, while the intensity of the other one remained almost the same. The reason is obvious, that the larger the size of the fluorescent volume, the greater the possibility that the excitation photons can enter it. The effect of altering absorption coefficient of the fluorescent volume is as shown in $(\mathrm{G})$. With the increase of the coefficient, the total energy in each plane also increases. This is because that the fluorescent volumes absorb more energy, thus emit more energy, when the absorption coefficient is higher. After all, no matter how the 
physical parameters change, the tendency of the photon distribution is constant, and that the peak positions correspond to the center of fluorescent volumes is always true. Such characteristics ensure the precise locating of the centers of fluorescent volumes under different circumstances.

Such law as we have acquired sheds light on the distribution of fluorescent photons, which is of great importance to the reconstruction of the FMT image. Additionally, the influence of other parameters, such as the size and forward coefficient to energy distribution can be obtained. Moreover, based on the simple exponential law equation, the quantitative energy distribution in an asymmetrical medium can be obtained by involving some correction factors. As a result, photon distribution patterns can be generalized as a priori knowledge, with which, the Monte Carlo reconstruction of FMT will converge more rapidly. As we all know, the Monte Carlo reconstruction for FMT could be simplified as the following steps: First, assumption for the model. Second, conduct Monte Carlo process and get a result. Third, compare the result with the real data and update the model. The initial parameters of the model will largely impact the convergence speed. However, we cannot give a reasonable initial model without any prior knowledge. Fortunately, through our discovery, this problem will be solved. As we mentioned in our manuscript, the distribution of the fluorescence photon can be delineated by a formula, based on which, the fluorescence photon distribution on the boundary could be easily obtained. This distribution is an approximate reflection of the real data detected by the detector. So, during the second step of Monte Carlo reconstruction method, we do not need any Monte Carlo process, we could just get an approximate distribution through the formulation and compared with the real distribution, if these matched well, then we get a reasonable initial model. This will largely improve the reconstruction speed.

\section{Conclusions}

In this paper, we presented a new Monte Carlo simulation program to explore the propagation of photons in fluorescent imaging. A series of simulations were designed to examine the relationship between the photon distribution, the parameters of the tissue, and the fluorescent volume. It is concluded that: (a) as the photon number increased, the energy distribution curve became smoother; (b) the fluorescence intensity decreased as the depth of the location of the fluorescent volume increased; (c) when the fluorescent volume was larger, the fluorescence intensity increased; and (d) as the anisotropy coefficient increased, more fluorescence photons were generated. Moreover, we compared our simulation results with those of MOSE, and it was found that our platform was robust. In the process, a shelter effect attracted our attention. Then, we identified the quantitative relationship between the photon distribution and the parameters of the tissue by using a control variable method; this proved that the photon distribution obeys an exponential law. In addition, in order to explore the effect of the phantom parameters on the exponential law, we designed a series of experiments, and the results demonstrated that the alteration of various parameters has little effect on the distribution trends, and only the relative value of fluorescence intensity changes. Thus the law can be used to detect the centers of different fluorescent volumes in various situations involving multiple fluorescent volumes. This will be a very important a priori knowledge. As mentioned before, this a priori knowledge will facilitate the convergence thus accelerate the speed in FMT reconstructions. If the center of the fluorescent volumes is known before the reverse process of the FMT image reconstruction, it is no doubt that the speed will be accelerated. In addition, because of the law we found, we can take full advantage of the discovery to create some database of the FMT forward process. The database is about relationship between the phantom characteristic and the energy distribution. The merit of this database is that it uses the formulation such as Eqs. (9) and (10) to describe the relationship rather than create a large database, as a result this will be very convenient. Moreover, since our Monte Carlo method not only focuses on the FMT forward problem, but it also takes incident light, such as CT ray into consideration. Therefore, energy distribution of X-ray attenuation can be explored, as a result, we can know the X-ray distribution inside the phantom and an attenuation correction database similar to the FMT energy distribution database can be constructed based on the Monte Carlo method as well. 


\section{References}

1. J. R. Lakowicz, Principles of Fluorescence Spectroscopy, Springer (2009).

2. R. Weissleder and V. Ntziachristos, "Shedding light onto live molecular targets," Nat. Med. 9, 123-128 (2003).

3. Y. Zhang, Q. Xu, J. Li, S. Tang and X. Zhang, "In vivo simulation environment for fluorescence molecular tomography using Monte Carlo method," in Int. Conf. Optical Instrument and Technology, 715607-1-715607-6 (2008).

4. R. Weissleder and U. Mahmood, "Molecular imaging," Radiology 219, 316-333 (2001).

5. R. G. Blasberg and J. Gelovani-Tjuvajev, "Molecular-genetic imaging," J. Cell. Biochem. 87, 172-183 (2002).

6. B. W. Rice, M. D. Cable and M. B. Nelson, "In vivo imaging of light-emitting probes," J. Biomed. Optics 6, 432-440 (2001).

7. J. Chang, H. L. Graber and R. L. Barbour, "Luminescence optical tomography of dense scattering media," JOSA A 14, 288-299 (1997).

8. E. E. Graves, J. Ripoll, R. Weissleder and V. Ntziachristos, "A submillimeter resolution fluorescence molecular imaging system for small animal imaging," Med. Phys. 30, 901 (2003).

9. H. He, L. Zhang, F. Gao, Z. Ma, H. Zhao and J. Jiang, "An analytic reflection method for timedomain fluorescence diffuse optical tomography based on a generalized pulse spectrum technique," Biomedical Optics (BiOS), pp. 68500M-68500M-8 (2008).

10. S. T. Flock, M. S. Patterson, B. C. Wilson and D. R. Wyman, "Monte Carlo modeling of light propagation in highly scattering tissues. I. Model predictions and comparison with diffusion theory," IEEE Trans. Biomed. Eng. 36, 1162-1168 (1989).

11. L. Wang, S. L. Jacques and L. Zheng, "MCMLMonte Carlo modeling of light transport in multilayered tissues," Computer methods and programs in biomedicine, Vol. 47, pp. 131-146, 1995.

12. A. T. Kumar, S. B. Raymond, A. K. Dunn, B. J. Bacskai and D. A. Boas, "A time domain fluorescence tomography system for small animal imaging," IEEE Trans. Med. Imaging 27, 1152-1163 (2008).

13. X. Zhang, C. Badea, M. Jacob and G. A. Johnson, "Development of a noncontact 3-D fluorescence tomography system for small animal in vivo imaging," SPIE BiOS: Biomedical Optics, 71910D-71910D-10 (2009).

14. G. Quan, H. Gong, Y. Deng, J. Fu and Q. Luo, "Monte Carlo-based fluorescence molecular tomography reconstruction method accelerated by a cluster of graphic processing units," J. Biomed. Optics 16, 026018-1-026018-8 (2011).

15. H. Li, J. Tian, F. Zhu, W. Cong, L. V. Wang, E. A. Hoffman et al., "A mouse optical simulation environment (MOSE) to investigate bioluminescent phenomena in the living mouse with the Monte Carlo method," Acad. Radiol. 11, 1029-1038 (2004).

16. C.-L. Tsai, J.-C. Chen and W.-J. Wang, "Near-infrared absorption property of biological soft tissue constituents," J. Med. Biol. Eng. 21, 7-14 (2001).

17. V. V. Tuchin, "Light scattering study of tissues," Phys. - Uspekhi 40, 495 (1997).

18. S. Tang, H. Yu and X. Mou, "Analytic simulation scheme for X-ray projections based on Physics Model," Optics 63 Photonics, pp. 63181T-1-63181T9 (2006). 